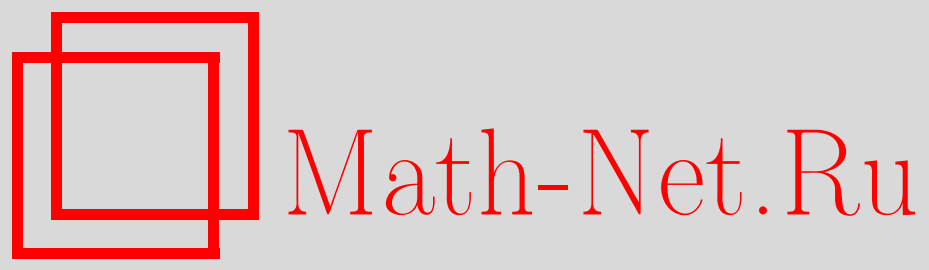

Н. А. Качановский, Г. Ф. Ус, Биортогональные системы Аппеля в анализе на дуально-ядерных пространствах, Функи. анализ и его прил., 1998, том 32, выпуск 1, 69-72

DOI: https://doi.org/10.4213/faa401

Использование Общероссийского математического портала MathNet.Ru подразумевает, что вы прочитали и согласны с пользовательским соглашением http://www . mathnet.ru/rus/agreement

Параметры загрузки:

IP: 34.239 .49 .27

26 апреля 2023 г., $17: 02: 56$

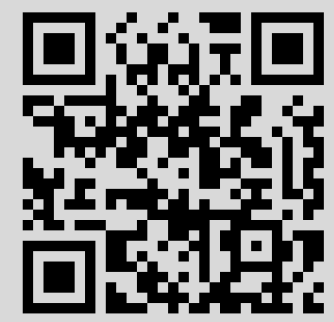




\title{
Биортогональные системы Аппеля в анализе на дуально-ядерных пространствах
}

\author{
(C) 1998. Н. А. КАЧАновСКий, Г. Ф. УС
}

Биортогональный анализ относительно аналитических мер $\mu$ на дуальноядерном пространстве $N^{\prime}$ построен в [1-4] на основе биортогонального разложения пространства $L_{2}\left(N^{\prime}, \mu\right)$ по системе $\mathbf{P}^{\mu}$ полиномов Аппеля на $N^{\prime}$ и двойственной системе обобщенных функций $\mathbf{Q}^{\mu}$. $\mathrm{B}[5,6]$ установлено, что подход из [1-4] может быть широко обобшен, если заменить экспоненты характерами $L_{1}$-гипергруппы, а обычный сдвиг - обобщенным. Отметим, что, согласно терминологии, принятой в классической теории полиномов Аппеля (см., например, [7]), система $\mathbf{P}^{\mu}$ из [1-4] состоит из полиномов Аппеля на $N^{\prime}$, порожденных голоморфной функцией $1 / l_{\mu}$, где $l_{\mu}$ - преобразование Лапласа меры $\mu$.

В настоящей заметке для построения анализа на $N^{\prime}$ применяется система полиномов Аппеля $\mathbf{P}^{A}$, порожденная произвольной голоморфной в нуле функцией $A$. В итоге получается более гибкая (в смысле приложений), чем в [2-4], шкала функциональных пространств, охватывающая пространства из [2-4] и сохраняющая все преимушества биортогонального подхода. Кроме того, примененная система $\mathbf{P}^{A}$ позволяет обобщить на бесконечномерный случай классический метод решения дифференциальных уравнений разложением в ряды по полиномам Аппеля [7].

1. Пусть $\mathscr{H}$ - вещественное сепарабельное гильбертово пространство, $N$ - сепарабельное ядерное пространство Фреше, плотно и непрерывно вложенное в $\mathscr{H}$, и $N^{\prime}$ - пространство, сопряженное к $N$ относительно $\mathscr{H}$. Тогда (в силу ядерности) $N=\operatorname{pr} \lim _{p \in \mathbb{N}} \mathscr{H}_{p}, N^{\prime}=$ ind $\lim _{p \in \mathbb{N}} \mathscr{H}_{-p}$, где $\mathscr{H}_{p}$, $p \in \mathbb{Z},-$ гильбертовы пространства, причем $\mathscr{H}_{p+1}$ вложено в $\mathscr{H}_{p}$ оператором типа Гильберта-Шмидта для любого $p \in \mathbb{Z}$ и $\mathscr{H}_{-p}$ - негативное пространство цепочки $\mathscr{H}_{-p} \supseteq \mathscr{H}_{2} \mathscr{H}_{p}$. Обозначим через $|\cdot|_{p}$ норму в $\mathscr{H}_{p}$, а через $\langle\cdot, \cdot\rangle$ - спаривание между $N^{\prime}$ и $N$, задаваемое расширением скалярного произведения в $\mathscr{H}$, и сохраним эти обозначения для тензорных степеней пространств и комплексификаций.

Пусть $N_{\mathbb{C}}$ - комплексификация пространства $N$. Обозначим через $\operatorname{Hol}_{0}\left(N_{\mathbb{C}}\right)$ алгебру ростков голоморфных в нуле функций $A: N_{\mathbb{C}} \rightarrow \mathbb{C}$.

Пусть $\mu$ - вероятностная мера на $\left(N^{\prime}, \mathscr{B}\left(N^{\prime}\right)\right)$, такая, что 1$)$ ее преобразование Лапласа $l_{\mu}(\cdot):=\int_{N^{\prime}} \exp \{\langle x, \cdot\rangle\} d \mu(x)$ принадлежит $\operatorname{Hol}_{0}\left(N_{\mathbb{C}}\right)$; $2)$ если $\varphi-$ непрерывный на $N^{\prime}$ полином, то $\varphi=0(\bmod \mu)$ влечет за собой $\varphi \equiv 0$. Совокупность таких мер, называемых аналитическими невырожденными мерами, обозначим через $\mathscr{M}_{a}\left(N^{\prime}\right)$.

2. Пусть $a \in \operatorname{Hol}_{0}(\mathbb{C}), a(0) \neq 0$. Положим $e_{a}(t ; x):=a(t) \exp \{x t\}, t, x \in \mathbb{C}$. В силу голоморфности функции $e_{a}$ представим ее в виде $\sum_{n=0}^{\infty} \frac{1}{n !} t^{n} P_{n}^{a}(x)$, где $P_{n}^{a}(x), n \geqslant 0$, - полином $n$-й степени. Полиномы $P_{n}^{a}(\cdot), n \geqslant 0$, называются 
полиномами Аппеля, порожденными функчией $a$, и $e_{a}-$ их производящая функция [7].

Введем бесконечномерный аналог системы $\left\{P_{n}^{a}(\cdot), n \geqslant 0\right\}$. Для $A \in$ $\operatorname{Hol}_{0}\left(N_{\mathbb{C}}\right), A(0) \neq 0$, функция $e_{A}(\theta ; z):=A(\theta) \exp \{\langle z, \theta\rangle\}, \theta \in N_{\mathbb{C}}, z \in N_{\mathbb{C}}^{\prime}$, допускает представление

$$
e_{A}(\theta, z)=\sum_{n=0}^{\infty} \frac{1}{n !}\left\langle P_{n}^{A}(z), \theta^{\otimes n}\right\rangle,
$$

где $P_{n}^{A}(z) \in N_{\mathbb{C}}^{\prime \widehat{\otimes} n}, n \geqslant 0$, а $\widehat{\otimes}$ - симметрическое тензорное произведение. Систему $\mathbf{P}^{A}:=\left\{\left\langle P_{n}^{A}(\cdot), \varphi^{(n)}\right\rangle: \varphi^{(n)} \in N_{\mathbb{C}}^{\widehat{\otimes} n}, n \in \mathbb{Z}_{+}\right\}$полиномов на $N^{\prime}$ назовем системой полиномов Аппеля, порожденных функцией $A$. Заметим, что при $A(\theta)=1 / l_{\mu}(\theta), \mu \in \mathscr{M}_{a}\left(N^{\prime}\right)$, система $\mathbf{P}^{A}$ изучена в [3].

Положим

$$
\begin{gathered}
\left(\mathscr{H}_{p}\right)_{q, A}^{1}:=\left\{\varphi(x)=\sum_{n=0}^{\infty}\left\langle P_{n}^{A}(x), \varphi^{(n)}\right\rangle:\|\varphi\|_{p, q, A}^{2}:=\sum_{n=0}^{\infty}(n !)^{2} 2^{n q}\left|\varphi^{(n)}\right|_{p}^{2}<\infty\right\}, \\
(N)_{A}^{1}:=\operatorname{pr}_{p, q \in \mathbb{N}}\left(\mathscr{H}_{p}\right)_{q, A}^{1} .
\end{gathered}
$$

В [3] доказано, что пространство основных функций $(N)_{1 / l_{\mu}}^{1}$ не зависит от $\mu \in \mathscr{M}_{a}\left(N^{\prime}\right)$ и совпадает с пространством $(N)^{1}$ сужений на $N^{\prime}$ целых на $N_{\mathbb{C}}^{\prime}$ функций первого порядка роста минимального типа. Аналогичная инвариантность имеется и для пространств $(N)_{A}^{1}$ (см. теорему 1$)$.

Теорема 1. Для любой функиии $A \in \operatorname{Hol}_{0}\left(N_{\mathbb{C}}\right), A(0) \neq 0,(N)_{A}^{1} u(N)^{1}$ совпадают как топологические пространства.

Для доказательства $x^{\otimes m}, x \in N^{\prime}$, выражаются через $P_{n}^{A}(x), n \leqslant m$, и используются неравенства Коши для голоморфных функщий на $N_{\mathbb{C}}^{\prime}$.

3. Пусть $a(t)=\sum_{n=0}^{\infty} \frac{1}{n !} a_{n} t^{n}, a_{0} \neq 0, t \in \mathbb{C}$, - голоморфная в нуле функция. Рассмотрим дифференциальное уравнение (бесконечного, вообще говоря, порядка) $a(D) y=f$. Если $f(t)=\sum_{n=0}^{\infty} f_{n} t^{n}$ - целая функция, то решение этого уравнения имеет вид $y(t)=\sum_{n=0}^{\infty} f_{n} P_{n}^{1 / a}(t)$, где $P_{n}^{1 / a}(\cdot)$, $n \geqslant 0$, - полиномы Аппеля, порожденные функцией $1 / a \in \operatorname{Hol}_{0}(\mathbb{C})$ (см., например, [7]).

Этот метод решения дифференциальных уравнений допускает обобщение на бесконечномерный случай. А именно, определим дифференциальный оператор $\left\langle\Phi^{(n)}, D^{\otimes n}\right\rangle$ порядка $n$ с постоянным коэффициентом $\Phi^{(n)} \in N_{\mathbb{C}}^{\prime \widehat{\otimes} n}$, полагая

$$
\left\langle\Phi^{(n)}, D^{\otimes n}\right\rangle\left\langle x^{\otimes m}, \varphi^{(m)}\right\rangle:=1_{\{m \geqslant n\}} \frac{m !}{(m-n) !}\left\langle x^{\otimes(m-n)} \widehat{\otimes} \Phi^{(n)}, \varphi^{(m)}\right\rangle
$$

$\left(1_{\{m \geqslant n\}}\right.$ - индикатор множества $\left.\{m \geqslant n\}\right)$ и продолжая его по линейности и непрерывности с мономов на $(N)^{1}$.

Пусть $\tilde{A}(\theta)=\sum_{n=0}^{\infty} \frac{1}{n !}\left\langle\tilde{A}_{n}, \theta^{\otimes n}\right\rangle, \tilde{A}_{0} \neq 0,-$ голоморфная в $0 \in N_{\mathbb{C}}$ функция (из (1) при $A=\tilde{A}$ и $z=0$ следует, что $\left.\tilde{A}_{n}=P_{n}^{\tilde{A}}(0)\right)$. Рассмотрим 
дифференциальное уравнение бесконечного, вообще говоря, порядка

$$
\tilde{A}(D) y \equiv \sum_{n=0}^{\infty} \frac{1}{n !}\left\langle\tilde{A}_{n}, D^{\otimes n}\right\rangle y=f .
$$

Теорема 2. Уравнение (3) корректно разрешимо в $(N)^{1}$. При этом, если $f(x)=\sum_{n=0}^{\infty}\left\langle P_{n}^{A}(x), \varphi^{(n)}\right\rangle, \operatorname{mo} y(x)=\sum_{n=0}^{\infty}\left\langle P_{n}^{A / \tilde{A}}(x), \varphi^{(n)}\right\rangle-$ его решение.

Это следует из соотношения $\tilde{A}(D)\left\langle P_{n}^{A}(x), \varphi^{(n)}\right\rangle=\left\langle P_{n}^{A \tilde{A}}(x), \varphi^{(n)}\right\rangle$, проверяемого непосредственным вычислением, и теоремы 1.

ЗАмечАниЕ 1. Оператор (2) можно продолжить по непрерывности на пространство $\left(\mathscr{H}_{p}\right)_{q, A}^{1}$, где $p$ больше или равно числа $p^{\prime}$, такого, что $\Phi^{(n)} \in$ $\mathscr{H}_{-p^{\prime}, \mathbb{C}}^{\widehat{\otimes} n}$, а $q>1$. При этом можно показать, что если $f \in\left(\mathscr{H}_{p}\right)_{q, A}^{1}$ для достаточно больших $p$ и $q$, то решение $y$ уравнения $(3)$ принадлежит $\left(\mathscr{H}_{p}\right)_{q, A / \tilde{A}}^{1} \subseteq$ $\left(\mathscr{H}_{p}\right)_{q-1, A}^{1}$.

ЗАмЕЧАНИЕ 2. Из теоремы 2 следует, что оператор $\tilde{A}(D)$ осуществляет изометрический изоморфизм между пространствами $\left(\mathscr{H}_{p}\right)_{q, A / \tilde{A}}^{1}$ и $\left(\mathscr{H}_{p}\right)_{q, A}^{1}$.

4. Пусть $\mu \in \mathscr{M}_{a}\left(N^{\prime}\right)$. Построим двойственную к $\mathbf{P}^{A}$ относительно $L_{2}\left(N^{\prime}, \mu\right)$ систему $\mathbf{Q}_{\mu}^{A}$ обобшенных функций. Для этого рассмотрим цепочку $\mathscr{P}_{\mu}^{\prime} \supseteq L_{2}\left(N^{\prime}, \mu\right) \supseteq \mathscr{P}$, где $\mathscr{P}$ - пространство непрерывных полиномов на $N^{\prime}$; через $\langle\langle\Phi, \varphi\rangle\rangle$ обозначим действие обобшенной функции $\Phi \in \mathscr{P}_{\mu}^{\prime}$ на основную $\varphi \in \mathscr{P}$. Пусть $A \in \mathrm{Hol}_{0}\left(N_{\mathbb{C}}\right), A(0) \neq 0$. Положим

$$
Q_{m}^{\mu, A}\left(\Phi^{(m)} ; \cdot\right):=\sum_{k=0}^{\infty} \frac{1}{k !}\left(\left\langle\Phi^{(m)} \widehat{\otimes} B_{k}, D^{\otimes(m+k)}\right\rangle^{*} 1\right)(\cdot), \quad \Phi^{(m)} \in N_{\mathbb{C}}^{\prime \widehat{\otimes} m},
$$

где $B_{k} \in N_{\mathbb{C}}^{\prime \widehat{\otimes} k}$ берется из разложения $1 /\left(A(\theta) l_{\mu}(\theta)\right)=\sum_{k=0}^{\infty} \frac{1}{k !}\left\langle B_{k}, \theta^{\otimes k}\right\rangle$, a оператор $\left\langle\Phi^{(m)}, D^{\otimes m}\right\rangle^{*} \in \mathscr{L}\left(\mathscr{P}_{\mu}^{\prime}\right)$ сопряжен к $(2)$ относительно $L_{2}\left(N^{\prime}, \mu\right)$. Соотношение (4) определяет обобщенную функцию из $\mathscr{P}_{\mu}^{\prime}$, поскольку для полинома $\varphi(x)=\sum_{k=0}^{n}\left\langle x^{\otimes k}, \varphi^{(k)}\right\rangle$ лишь конечное число слагаемых в спаривании $\left\langle\left\langle Q_{m}^{\mu, A}\left(\Phi^{(m)} ; \cdot\right), \varphi(\cdot)\right\rangle\right\rangle$ отлично от нуля. Более того, справедлив следующий результат (совпадающий в случае $A=1 / l_{\mu}$ с доказанным в $[2,3]$ ).

Теорема 3. Система $\mathbf{Q}_{\mu}^{A} \subset \mathscr{P}_{\mu}^{\prime}$ элементов вида (4) биортогональна $\kappa$ $\mathbf{P}^{A}$ в том смивсле, что $\left\langle\left\langle Q_{m}^{\mu, A}\left(\Phi^{(m)} ; \cdot\right),\left\langle P_{n}^{A}(\cdot), \varphi^{(n)}\right\rangle\right\rangle\right\rangle=\delta_{n m} n !\left\langle\Phi^{(n)}, \varphi^{(n)}\right\rangle$. Любой элемент $\Phi \in \mathscr{P}_{\mu}^{\prime}$ единственным образом представим в виде

$$
\Phi(\cdot)=\sum_{m=0}^{\infty} Q_{m}^{\mu, A}\left(\Phi^{(m)} ; \cdot\right), \quad \Phi^{(m)} \in N_{\mathbb{C}}^{\prime \widehat{\otimes} m}
$$

Эта теорема доказывается прямым вычислением, причем ядра $\Phi^{(m)}$ из $(5)$ определяются соотношением

$$
\left\langle\Phi^{(m)}, \varphi^{(m)}\right\rangle:=\left\langle\left\langle\Phi(\cdot),\left\langle P_{m}^{A}(\cdot), \varphi^{(m)}\right\rangle\right\rangle, \quad \varphi^{(m)} \in N_{\mathbb{C}}^{\widehat{\otimes} m} .\right.
$$


В терминах системы $\mathbf{Q}_{\mu}^{A}$, подобно тому, как это делается в $[2,3]$, описываются сопряженные к $\left(\mathscr{H}_{p}\right)_{q, A}^{1}$ и $(N)_{A}^{1}=(N)^{1}$ относительно $L_{2}\left(N^{\prime}, \mu\right)$ пространства. А именно, пусть $\left(\mathscr{H}_{-p}\right)_{-q, A, \mu}^{-1} \subseteq \mathscr{P}_{\mu}^{\prime}-$ пространство обобщенных функций $\Phi \in \mathscr{P}_{\mu}^{\prime}$, для которых $\|\Phi\|_{-p,-q, A, \mu}^{2}:=\sum_{m=0}^{\infty} 2^{-q m}\left|\Phi^{(m)}\right|_{-p}^{2}<\infty$, где $\Phi^{(m)} \in N_{\mathbb{C}}^{\prime \widehat{\otimes} m}$ - ядра из представления (5). Тогда для достаточно больших p и q пространство $\left(\mathscr{H}_{-p}\right)_{-q, A, \mu}^{-1}$ является сопряженныл $\kappa\left(\mathscr{H}_{p}\right)_{q, A}^{1}$ относительно $L_{2}\left(N^{\prime}, \mu\right)$, a $(N)_{\mu}^{-1}:=\operatorname{ind} \lim _{p, q \in \mathbb{N}}\left(\mathscr{H}_{-p}\right)_{-q, A, \mu}^{-1}$ сопряжено $\kappa(N)^{1}$.

Отметим, что на $(N)_{\mu}^{-1}$ можно рассматривать дифференциальные уравнения, сопряженные к уравнениям вида (3), для которых справедлив аналог теоремы 2.

Авторы благодарны Ю. М. Березанскому, Ю. Л. Далецкому и Ю. Г. Кондратьеву за полезные обсуждения и внимание к работе.

\section{ЛитеРАтУРА}

1. Далецкий Ю. Л. Функц. анализ и его прил., 25, вып. 2, 68-70 (1991). 2. Albeverio S., Daletzky Yu., Kondratiev Yu., Streit L. J. Funct. Anal., 138, No. 2, 311-350 (1996). 3. Kondratiev Yu., Streit L., Westerkamp W., Yan J. Generalized functions in infinite dimensional analysis, IIAS report No. 1995-002, 1995. 4. Us G. F. Methods Funct. Anal. Topol., 1, No. 1, 93-108 (1995). 5. Березанский Ю. М., Кондратьев Ю. Г. Функц. анализ и его прил., 29, вып. 3, 51-55 (1995). 6. Березанский Ю. М. Функц. анализ и его прил., 30, вып. 4, 61-65 (1996). 7. Boas R. P., Buck R. C. Polynomial expansions of analytic functions. Springer, Berlin, 1964.

Киевский политехнический институт

Киевский университет им. Тараса Шевченко
Поступило в редакцию 27 сентября 1995 г. В переработанном виде 23 мая 1997 г.

УДК 517.9

\section{Базисные функции, связанные с двумерной системой Дирака}

(C) 1998. О. М. КИСелЕВ

В работе построен специальный базис в пространстве гладких интегрируемых функций двух вещественных переменных. Базисные функции связаны с решением двумерной системы Дирака

$$
\left(\begin{array}{cc}
\partial_{\bar{z}} & 0 \\
0 & \partial_{z}
\end{array}\right) \varphi=\frac{1}{2}\left(\begin{array}{cc}
0 & q \\
-\bar{q} & 0
\end{array}\right) \varphi,\left.\quad E(-k z) \varphi\right|_{|z| \rightarrow \infty}=I .
$$

Здесь $z, k$ принадлежат $\mathbb{C}$, черта означает комплексное сопряжение, $E(k z)=$ $\operatorname{diag}(\exp (k z), \exp (\overline{k z})), I$ - единичная матрица.

* Работа выполнена при поддержке РФФИ (грант 97-01-00459) и Международного научного фонда (грант MNB300). 